\title{
Room-temperature superconductivity and quantum physics
}

\author{
Armen Gulian - Deborah Van Vechten - Emmanuele Cappelluti • \\ Oleg Dolgov • Jeff Tollaksen • Gurgen Melkonyan • \\ Boris Gorshunov · Cristian Bourgeois
}

(C) Chapman University 2017

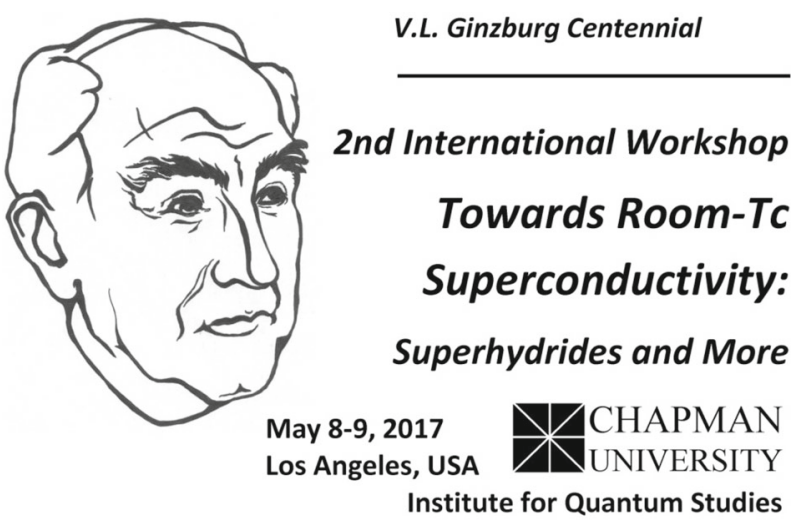

A. Gulian ( $\square)$

Chapman University, Burtonsville, MD, USA

e-mail: gulian@chapman.edu

D. V. Vechten

Office of Naval Research, Arlington, VA, USA

E. Cappelluti

Istituto di Struttura della Materia, CNR, Trieste, Italy

O. Dolgov

Max-Planck-Institut für Festkörperphysik, Stuttgart, Germany

O. Dolgov

P.N. Lebedev Physical Institute, Moscow, Russia

J. Tollaksen · C. Bourgeois

Chapman University, Orange, CA, USA

G. Melkonyan

GMLab, Quebec, Canada

B. Gorshunov

Moscow Institute of Physics and Technology (State University), Dolgoprudny, Moscow Region, Russia 
Superconductivity is one of the most complex topics in physics and achieving the goal of room-temperature superconductivity has been one of the most challenging pursuits of modern physics. Many dedicated scientists have spent substantial resources and published many articles in the effort to understand high-temperature superconductivity. Even though the ultimate goal has yet to be realized, there is optimism amongst the scientific community that such an understanding will come soon. The remarkable finding of superconductivity at 205K (and two million atmospheres pressure) by Dr. Eremets and his group increased confidence that room-temperature superconductivity can be expected in the not too distant future.

This volume contains selected papers following presentations at the Second International Workshop "Towards Room-Temperature Superconductivity: Superhydrides and More". This workshop commemorated the Centennial of V.L. Ginzburg, one of the greatest proponents of high-temperature superconductivity, and was organized by the Institute for Quantum Studies at Chapman University (Orange, California). About 50 researchers and scholars throughout the world gathered to discuss problems and achievements in the field, as well as to learn from each other. It is hard to overestimate the importance and foundational meaning of such meetings. The first one was organized by our Italian colleagues in Rome during 2016. We hope that this workshop will continue annually, thereby promoting research throughout the world.

The beauty at the heart of superconductivity is in its quantum nature. Underlying mechanisms for superconductivity are based on foundational quantum postulates. It is not surprising that the description of basic features of superconductors, as well as the prediction of novel superconductors requires the full spectrum of quantum fieldtheoretical methods, which demands mastering theoretical physics. Furthermore, high-level mastery of experimental physics is required when applying superconductivity to practical purposes. So, for now, superconductivity attracts a relatively small number of specialists to the field. However, this will change when superconductors become available at ambient temperatures and pressures. Society will then have more opportunities to appreciate its valuable role in improving daily life.

It is important to keep in mind that superconductors are quite unique in that they are macroscopic quantum objects. The fascination with quantum mechanics is already widespread throughout the general population. For example, when traveling from Los Angeles International airport to attend the Workshop, a lively conversation about quantum entanglement occurred between one of us and the very curious taxi driver. His bevy of questions was quite unexpected, and reflected the interest of a wider audience on fundamentals of the quantum world. It is hard to imagine the level of interest when people are actually able to hold in their hands lumps of these macroscopic envoys of the quantum world - superconductors alive at room temperature! Despite, or perhaps because of, the mystery surrounding any quantum object, many unusual applications of superconductors will be found. Replacement of the already ubiquitous transistors will make electronics smaller, more powerful, and much smarter, drastically altering modern technology. Most importantly, the limitations on high-power applications will become obstacles in the imagination only as schemes such as transferring solar energy from the Sahara Desert through trans-Atlantic superconducting cables become realistic.

For that to happen, researchers need to keep working, and working as relentlessly and productively as possible. We, the members of the organizing committee, would like to express gratitude to all participants of the Workshop. Below is the list of plenary talks which have been given at Chapman:

1. M. I. Eremets, News on 203 K superconductivity

2. L. Pietronero, Conventional/unconventional superconductivity in high pressure hydrides and beyond: Insights from theory and perspectives

3. B.M. Klein, Progress toward the discovery of a room-temperature superconductor: What drives the high $T_{c}$ of the superhydrides at high pressure, and where do we go from here?

4. D. A. Papaconstantopoulos, The electron-phonon coupling in light-element hydrides

5. A. Blom, What we can and cannot yet accomplish in search of novel superconductors with QuantumWise

6. F. Zhang, Possible superconductivity above ice point

7. L.Z. Deng, Possible interface superconductivity in rare-earth doped $\mathrm{CaFe}_{2} \mathrm{As}_{2}$ and undoped $\mathrm{CaFe}_{2} \mathrm{As}_{2}$

8. J. Xia, Towards room temperature $2 D$ superconductivity at magnetic-semimetal interface 
9. A.M. Gulian, Serendipitous vs. systematic search for room-temperature superconductivity

10. I. Božović, Following V. L. Ginzburg: on the road to room-temperature superconductivity

11. T. H. Geballe and J. Mannhart, Raising $T_{c}-$ A different method

12. P.M. Grant, Superconducting fluctuations in one-dimensional quasi-periodic "metallic" chains: The Little model of room temperature superconductivity embodied

13. L. Ortenzi, Tight binding model and channel like structures in $\mathrm{H}_{3} \mathrm{~S}$ and their implications for the superconducting pairing

14. M.V. Sadovskii, Electronic structure of FeSe monolayers: why $T_{c}$ is so high?

15. S.-H. S. Salk, Plausible room temperature superconducting phase pransitions based on holon-pair slave-boson theory of antiferromagnetic fluctuations

16. Q. Li, Chiral magnetic effect in condensed matter-A new route for non-dissipative charge transport at room temperature

17. P. Esquinazi, Evidence for superconductivity at room temperature at graphite interfaces

18. X.-J. Chen, Superconductivity above $120 \mathrm{~K}$ in polyparaphenylene oligomers

In addition, there were many posters. Poster presenters and plenary speakers contributed on equal footing to this peer-reviewed volume. We are grateful for their effort to communicate their findings and ideas with the wider audience of readers.

We also would like to express sincere gratitude to the sponsors of this year's Workshop: US Office of Naval Research, Oxford Instruments, Cryogenic LTD, Quantum Design, GloMo, and Chapman University.

Finally, we would like to express our gratitude to Sir Anthony Leggett, the Honorary Chair of the program committee, who shared in 2003 the Nobel Prize in physics with V.L. Ginzburg and A.A. Abrikosov. In his Nobel Lecture, he said:

"I got more and more intrigued by the conceptual formulations of quantum mechanics, and by the summer of 1972 had made a firm decision that I would abandon the sort of physics that gets published in Phys. Rev. B and devote myself full-time to foundational studies."

As we celebrate Tony's 80th birthday this year, we reflect on his many remarkable achievements throughout his career exploring both the topics of foundations of physics and superconductivity along with the relationships between them.

Armen Gulian, PhD, Quest Editor of this Volume (Chair of Program Committee)

Deborah Van Vechten, PhD (USA) (Member of Program Committee)

Emmanuele Cappelluti, PhD (Italy) (Member of Program Committee)

Oleg Dolgov, PhD (Germany) (Member of Program Committee)

Jeff Tollaksen, $\mathrm{PhD}$ (USA) (Member of Program Committee)

Gurgen Melkonyan, $\mathrm{PhD}$ (Canada) (Member of Program Committee)

Boris Gorshunov, $\mathrm{PhD}$ (Russia) (Member of Program Committee)

Cristian Bourgeois, Quest Technical Editor of This Volume (Chair of Local Organizing Committee) 